\title{
Juego online: tratamiento de un caso de adicción a apuestas deportivas
}

\section{Online gambling: A treatment for sports betting addiction}

\author{
Marta Marcos* \\ Universidad de Valencia, España
}

\section{Resumen}

Antedecentes: el juego de azar online (apuestas, juegos de casino, póquer, etc.) es una actividad que se está extendiendo mundialmente, incluso en los países en los cuales todavía no está legalizado. A pesar de que la situación en Latinoamérica es de lo más diversa, es probable que conforme se desarrolle y promocione el juego online aparezcan problemas de adicción, de forma similar a lo que está ocurriendo en España desde que este tipo de juegos se legalizó en 2011. De manera que la ciencia psicológica debe ir desarrollando programas de tratamiento que hagan frente a la nueva realidad. Objetivo: el artículo presenta el procedimiento seguido para el tratamiento de un joven con problema de adicción a las apuestas deportivas. Método: la propuesta de tratamiento tiene una orientación conductual-cognitiva, en la que se produjo descondicionamiento de las situaciones de juego, entrenamiento en habilidades para prevenir recaídas y superar situaciones de riesgo, así como información de los riesgos del juego, cambio de actitudes hacia las apuestas y promoción de un estilo de vida saludable incompatible con el juego excesivo. Resultados: se describen tanto las fases del tratamiento como el fundamento teórico de la propuesta de intervención. El paciente, con un diagnóstico de trastorno de juego grave, redujo los criterios de trastorno por juegos de apuesta, así como el Índice de Gravedad de Síntomas clínicos. Conclusiones: se ha desarrollado un protocolo de tratamiento de la adicción al juego online basado en los principios de la motivación y el aprendizaje que ha resultado ser eficaz en la reducción de la conducta de juego y la consolidación de un nuevo estilo de vida saludable.

Palabras clave: juego online; trastorno por juego de apuestas; tratamiento; adicción al juego; juventud; juego ético.

\section{Para citar este artículo:}

Marcos, M. (2020). Juego online: tratamiento de un caso de adicción a apuestas deportivas. Liberabit, 26(2), e399. https://doi.org/ 10.24265/liberabit.2020.v26n2.04

\begin{abstract}
Background: online gambling (bets, casino games, poker, etc.) is an activity that is spreading worldwide, even in countries where it is not yet legalized. Although the situation in Latin America is very heterogeneous, it is possible that, as online gambling is developed and promoted, addiction problems arise. This has already happened in Spain since the legalization of online gambling in 2011. Thus, psychology should be developing intervention programs to face this new scenario. Goal: this paper describes the treatment protocol for a young man with sports betting addiction. Method: the treatment proposal consisted in a cognitive behavioral therapy which focused on deconditioning of gambling situations; training of skills needed to prevent relapses and overcome risky situations; information on gambling risks; change of attitude towards gambling; and promotion of a healthy lifestyle incompatible with gambling addiction. Results: both the treatment phases and the theoretical basis of the intervention proposal are described. The patient, diagnosed with a severe gambling disorder, showed improvements in the gambling disorder criteria and the Symptom Severity Scale Score. Conclusions: a treatment protocol for online gambling addiction, which is based on the principles of motivation and learning, has been developed. This protocol has demonstrated to be effective in reducing gambling behavior and promoting a new healthy lifestyle.
\end{abstract}

Keywords: online gambling; gambling disorder; treatment; gambling addiction; youth; ethical gambling.

Este es un artículo Open Access publicado bajo la licencia Creative Commons Atribución 4.0 Internacional. (CC-BY 4.0)

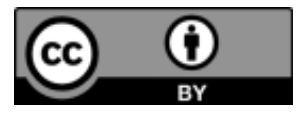

Universidad de San Martín de Porres, Lima - Perú http://ojs3.revistaliberabit.com 


\section{Introducción}

El término adicción comportamental, o no química, fue definido inicialmente por Isaac Marks (1990) en una editorial del British Journal of Psychology para referirse a un síndrome caracterizado por la necesidad de llevar a cabo una conducta de forma tal que resulta perjudicial para la persona. En tanto dicha conducta no se lleve a cabo, se produce un incremento en la tensión que se acumula hasta que se ejecuta el comportamiento y dicha tensión desaparece. Este impulso está inducido por claves externas condicionadas que pueden establecer un condicionamiento secundario con otros estímulos internos, tanto viscerales como cognitivos o emocionales. Dicha conducta puede prevenirse estableciendo un adecuado control estimular y la reducción del impulso se puede llevar a cabo mediante extinción o descondicionamiento de las claves que la provocan, que son los procesos en los que se basa el tratamiento del juego patológico mediante el control de estímulo y exposición gradual con prevención de respuesta (Echeburúa et al., 1996). No obstante, la recompensa económica que a veces se produce, junto con las condiciones del propio juego, son fundamentales para entender que la conducta de jugar está sometida a un proceso de condicionamiento operante. De hecho, el juego electrónico está diseñado específicamente para provocar una conducta adictiva (Schüll, 2014) y, en este caso, las apuestas deportivas tienen los principales elementos estructurales para generarlas, principalmente, controlando el cociente de apuestas de forma que se ajuste a programas concurrentes de reforzamiento (Pierce \& Cheney, 2013) que induzcan elevadas tasas de conducta.

En lo que se refiere a las clasificaciones de psicopatología, el juego patológico era considerado como un trastorno del control del impulso tanto en el DSM-III (American Psychiatric Association, 1980) como en el CIE-9 (Organización Mundial de la Salud [OMS], 1977). Si bien desde muy pronto, tanto clínicos como científicos reivindicaron que el juego patológico podría clasificarse más adecuadamente como un trastorno por dependencia que del control de impulsos. Finalmente, tanto en el DSM-5 (American Psychiatric Association, 2013) como en el CIE-11 (OMS, 2018a), el trastorno por juegos de apuesta se clasifica en la misma categoría que los trastornos por consumo de sustancias, entendiéndose, de esta manera, que se trata de una adicción comportamental. En la clasificación de la OMS se distingue, además, entre los subtipos online y offline. Si bien es la actividad de apostar la que activa los mismos circuitos cerebrales de recompensa que las drogas (Goudriaan et al., 2004) y la sintomatología clínica es esencialmente la misma que la de cualquier adicción (American Psychiatric Association, 2013), lo cierto es que las diferentes modalidades de juego tienen diferente potencial adictivo. De entre ellos, el juego online es más adictivo que las modalidades tradicionales de juego (Chóliz et al., 2019; McBride \& Derevensky, 2012), lo cual justifica la investigación y desarrollo de procedimientos de intervención específicos para estos trastornos. A su vez, asumir que el trastorno por juegos de apuesta online es una adicción comportamental tiene la ventaja de que provee de un marco conceptual no solo para entender la génesis y mantenimiento del problema, sino también para el diseño de procedimientos de tratamiento adaptados a esta nueva problemática.

El desarrollo de la electrónica y la aplicación de Internet a las actividades cotidianas han supuesto una auténtica revolución en la vida de las personas. Se trata de un fenómeno que está afectando en mayor o menor medida a gran parte de las sociedades y que no solamente tiene relevancia en la dimensión económica, sino que modifica sustancialmente la forma de comportarse y de relacionarnos. Parece indudable que las Tecnologías de la Información y Comunicación (TIC) han mejorado considerablemente nuestra calidad de vida y han permitido que personas con dificultades físicas, psicológicas o incluso económicas, puedan acceder a actividades y recursos que de otra forma era impensable que pudieran conseguir. Y ello gracias a las TIC. 
Estos avances tecnológicos, que sin duda son muy positivos y caracterizan la modernidad de los tiempos en los que tenemos la suerte de vivir, pueden acarrear riesgos reales si favorecen conductas disfuncionales o directamente patológicas, como es el caso del trastorno de juego. Y es que la aplicación de los desarrollos de la electrónica e Internet al caso del juego permiten que se modifiquen las condiciones estructurales de una actividad ya de por sí adictiva (American Psychiatric Association, 2013), incrementando el potencial de provocar el trastorno de juego (Chóliz et al., 2019; Griffiths \& Barnes, 2008; McBride \& Derevensky, 2012). Tanto es así que la última edición del manual de enfermedades de la OMS, el ICD-11, ya reconoce como entidad diagnóstica al trastorno de juego online (OMS, 2018b).

Las condiciones del juego que favorecen la aparición de conductas disfuncionales que pueden conducir a la aparición de un trastorno de juego online son de dos tipos: estructurales y ambientales. Algunas de las condiciones estructurales que favorecen la adicción a este tipo de juego son (Griffiths, 2003) la inmediatez del resultado de la apuesta, la accesibilidad con la que es posible jugar desde cualquier dispositivo electrónico con acceso a la red, la comodidad con la que se puede apostar o incluso la intimidad con la que es posible llevarla a cabo.

En lo que se refiere a las condiciones ambientales, están directamente relacionadas con la normativa de cada país y harían referencia a variables como la oferta de los juegos, la disponibilidad (cantidad de webs o locales dónde jugar online), la promoción o publicidad del juego, entre otros. En este sentido, se trata de variables que están relacionadas con el estatus legal del juego online. Sin embargo, podrían no depender exclusivamente de ello, puesto que se puede jugar a través de Internet en páginas webs localizadas en países (generalmente paraísos fiscales), a pesar de que no haya una regulación en el estado desde donde apuesta el jugador.
En Latinoamérica nos encontramos en una situación de lo más diversa respecto al juego online. Hay países en los que el juego está totalmente prohibido y no existe el juego tradicional ni online, como es el caso de Cuba. Así también, están los países en los que, a pesar de estar prohibido el juego, este se encuentra presente y operativo a través de webs internacionales, como es el caso de Ecuador. Otros países tienen autorizado el juego tradicional y expresamente prohibido el juego online, como en Uruguay; mientras que otros no lo tienen regulado, pero tampoco expresamente prohibido, como Paraguay o Perú; o lo tienen parcialmente en algunas partes de su territorio, como ocurre en Argentina. Finalmente, están los países que lo tienen totalmente autorizado, como es el caso de México o Colombia.

La autorización legal suele estar directamente relacionada con la promoción de esta actividad y con la generalización del juego online como una actividad cotidiana, especialmente, por parte de población juvenil y adolescente, que es el «nicho de mercado» al que se suelen dirigir las estrategias de promoción y marketing. Sin embargo, es posible jugar online en un país que no lo tenga autorizado, en unas condiciones muy parecidas a las que se pueden llevar a cabo en donde este sea legal: a través de páginas web localizadas en otros países del mundo. Esto tiene como consecuencia que el trastorno de juego online pueda aparecer en una sociedad donde, inclusive, este no esté legalizado. La legalización del juego online tiene como consecuencia la promoción y generalización de esta actividad y con ello, el incremento de trastornos de juego online, especialmente, en la población más joven.

En España se ha pasado de estar prohibidos la mayoría de juegos durante la dictadura franquista, a una de las legislaciones más permisivas y una oferta de juego de las más amplias del mundo (Chóliz \& Marcos, 2018). En lo que se refiere al juego online, las empresas llevan operando y publicitándose desde principios de 2000, si bien solo es legal desde la concesión de las licencias en julio de 2012. Desde 
entonces, la oferta de juegos en España, el gasto de los españoles en esta actividad y los indicadores de prevalencia de adicción al juego online han crecido exponencialmente. Al punto de que se está convirtiendo en un problema de salud pública y haciendo necesaria una intervención desde todos los ámbitos de la sociedad, pero especialmente desde los poderes públicos (Chóliz, 2018). Afortunadamente, la ciencia psicológica conoce desde hace tiempo principios y leyes relevantes que gobiernan el comportamiento, así como el papel de los procesos psicológicos implicados, lo cual facilita no solamente el conocimiento de nuevas realidades, sino también la aplicación de las necesarias técnicas y procedimientos de intervención para la modificación de conductas que pueden llegar a ser disfuncionales, desadaptativas o, directamente, patológicas, como es el caso de la adicción al juego online.

El presente trabajo describe un estudio de caso basado en un protocolo de tratamiento psicológico de adicción al juego online (Chóliz \& Marcos, 2020), adaptado y aplicado en todas sus fases a las características individuales del caso de adicción a apuestas deportivas que se describe; en concreto, apuestas de tenis. Se exponen las principales características del caso, así como las fases en las que se dividió el tratamiento de la adicción al juego online en el que se produjeron cambios que no solamente afectaron a la reducción de la conducta problemática, sino al propio proceso de dependencia y al funcionamiento personal y social del paciente. Dichos cambios se mantienen un año después de la finalización de la intervención.

\section{Estudio del caso}

Alberto (nombre ficticio) es un español de 23 años de edad, soltero y sin hijos. Actualmente, mantiene una relación a distancia con una joven de Barcelona con la que convivía antes de trasladarse a casa de sus padres en Valencia. Su nivel formativo es alto, de profesión árbitro, habiendo terminado el ciclo superior de técnico en Educación Física. A su llegada a la consulta estaba en situación de desempleo, pero al finalizar el tratamiento ya se encontraba trabajando en actividades relacionadas con su formación. Hasta volver de Barcelona a Valencia trabajó como dependiente en una cadena de supermercados.

El paciente regresa a casa de sus padres para iniciar un tratamiento por su problema con las apuestas de tenis y recibir contención familiar, siendo su novia la que llama a la madre de Alberto para explicarle la situación. Respecto al motivo de consulta, es él mismo quien contacta con la Unidad de Investigación de Juego y Adicciones Tecnológicas tras haber conocido el recurso de tratamiento que se estaba llevando a cabo. Aunque viene voluntariamente, refiere que lo hace «por insistencia de su novia y su madre».

En la primera sesión acude con la madre y cuando se le pide que describa su problema, Alberto presenta taquilalia: refiere que tiene un problema con las apuestas deportivas en el tiempo que ha estado viviendo solo en Barcelona y reconoce que «trabajaba gratis porque lo gastaba todo, y más, en apostar». Describe una evolución del problema de juego con falta de conciencia de ser una enfermedad hasta que la deuda ascendió a 6000 euros, momento en el que se da cuenta que ha perdido el control. Decide no contarlo y logra resolver la mitad de la deuda por sí mismo; sin embargo, no consigue parar y continúa apostando. Actualmente las deudas pendientes ascienden a 3000 euros que corresponden a préstamos adquiridos en entidades bancarias con el fin de seguir jugando y recuperar lo perdido. Su gasto en juego diario era aproximadamente de unos 120 euros diarios.

Alberto, actualmente, es consciente de su problema, presenta motivación para el cambio y se encuentra en la fase de acción del modelo transteórico del cambio en adicciones (Prochaska et al., 1992). Ya ha comenzado con pequeñas pautas de control de estímulos acordadas con la madre y supervisadas por esta que es quien le proporciona el apoyo y la supervisión necesaria. Puesto que el 
paciente ya ha comenzado a realizar pequeños cambios y presenta criterios de intención y conducta combinados, se refuerzan los pequeños cambios que ha dado y se inicia el tratamiento psicológico de la adicción al juego online. Esto sin dejar de evaluar en todo el proceso del tratamiento cómo es su disposición al cambio para, en el caso que haya detección de recaída motivacional, evitar la recaída conductual y modificar el plan establecido para mantener la abstinencia y conseguir los objetivos marcados.

En la primera sesión, Alberto firmó el consentimiento informado y se le explicó el compromiso de la terapeuta en base al código deontológico, asegurando en todo momento la confidencialidad de la información y el secreto profesional.

\section{Antecedentes}

En las entrevistas iniciales se determinó que el paciente no había sufrido ninguna pérdida importante u estresor reciente que pudiera haber agravado el problema con las apuestas. No presentó consumo de drogas y nunca antes había estado en tratamiento psicológico ni psiquiátrico, siendo esta situación la primera vez que realiza una consulta a un profesional de la salud mental. En lo que se refiere a los intentos por sí mismo de abandonar el juego han sido tres, pero sin éxito, llegando a permanecer un tiempo máximo de apenas tres meses sin jugar. No existen antecedentes familiares por problemas con el juego. Respecto al apoyo social, cuenta con la ayuda de sus padres y con algún amigo en quien puede confiar en los momentos más difíciles. En relación a la violencia de género, afirma no haber sufrido malos tratos físicos o psicológicos y tampoco haberlos infringido él mismo.

\section{Método}

\section{Procedimiento}

El tratamiento se ofreció como recurso terapéutico por parte de la Universidad de Valencia, desde la Unidad de Investigación «Juego y adicciones tecnológicas». El paciente fue derivado desde los servicios sociosanitarios por su problema con las apuestas deportivas de tenis.

\section{Diseño}

Según la clasificación de Montero y León (2005), se empleó un diseño de caso único AB (Barlow \& Hersen, 1988), siendo la intervención de carácter individual y grupal con seguimientos (1 - 3 - 12 - 24 meses) después del alta terapéutica.

La intervención terapéutica siguió las fases indicadas en el protocolo de tratamiento desarrollado por Chóliz y Marcos (2020), que consta de las siguientes etapas:

1. Evaluación y diagnóstico. Además de la entrevista clínica, el análisis funcional de la conducta y el estudio del patrón de juego se le administraron los siguientes instrumentos:

- NODS (Gerstein et al., 1999). Se trata de un cuestionario que consta de 17 ítems y procede de una entrevista semiestructurada para el diagnóstico de trastorno de juego basado en los criterios del DSM-IV-TR (American Psychiatric Association, 2000). El rango de las puntuaciones oscila entre 0 y 9 . La corrección del NODS se adaptó al actual DSM-5 eliminando el criterio correspondiente a la obtención de dinero de forma ilegal para poder seguir jugando para el diagnóstico de trastorno de juego. El análisis de la fiabilidad de la prueba en el estudio de Chóliz y Marcos (2020) fue de $\alpha=.87$.

- Síntomas clínicos y psicopatológicos (SCL90; Derogatis \& Cleary, 1977). Se utilizó la versión española de Robles, Andreu y Peña (2002). Se trata de una escala que evalúa nueve 
síntomas clínicos y tres indicadores de malestar. Las dimensiones sintomatológicas primarias son somatización, obsesividadcompulsividad, sensibilidad interpersonal, depresión, ansiedad, hostilidad, ansiedad fóbica, ideación paranoide y psicoticismo. El análisis de la fiabilidad a través de la consistencia interna de las escalas oscila desde $\alpha=.69$ (ansiedad fóbica) a $\alpha=.85$ (somatización). Los tres índices globales que proporcionan una medida de distrés son los siguientes: Índice de Sintomatología General (GSI, General Symptom Index), Índice de Alteración de Síntomas Positivos (PSDI, Positive Symptom Distress Index) y el Total de Síntomas Positivos (PST, Positive Symptom Total).

Se puso especial énfasis en la motivación para el cambio como un estilo de relación entre el paciente y el terapeuta que debe acompañar transversalmente todo el tratamiento y que no debe confundirse con la técnica de la entrevista motivacional (EM; Miller \& Rollnick, 1991). Esta última solo se utilizaría en aquel paciente que no presentara conciencia de enfermedad o se encontrara en estadios previos a la preparación para poder iniciar el plan de tratamiento establecido con un mínimo de garantías. En las consultas iniciales se ofreció contención y se favoreció el establecimiento del vínculo terapéutico dentro un clima de empatía y cordialidad exento de juicios para asegurar la confianza que nos asegurara poder conocerlo y su adherencia al tratamiento. Al mismo tiempo, se suministró información acerca del proceso adictivo y la adicción, favorecida por las características estructurales de los juegos (inmediatez, atractivo, programa de reforzamiento de razón variable, etc.). Todos estos factores hacen que el jugador juegue cada vez más, lo cual es un peligro. Esto es debido a que el cociente de apuestas está diseñado mediante algoritmos que hacen que la esperanza matemática siempre sea positiva para la empresa que organiza el juego y, por lo tanto, negativa para el jugador. Es decir, que a la larga el jugador pierde más de lo que apuesta. El objetivo fue favorecer el cambio de actitudes hacia el juego, basándonos en los principios del juego ético (Chóliz, 2018), que postulan que el juego es una actividad potencialmente adictiva que, a pesar de ello, está muy promocionada en nuestra sociedad actual, puesto que es muy rentable para algunas empresas. Tan es así que la adicción hace que los jugadores apuesten cada vez más y con más frecuencia, agravando el problema que no solo es económico, sino, principalmente, de salud.

También se realizó el encuadre y la firma del contrato terapéutico que recogió el compromiso de Alberto, previamente acordado y consensuado sobre las pautas a seguir para llevar a cabo la intervención (ver Figura 1).

Se indicaron, específicamente, las tareas que se le requerían: a) cumplimentación del registro de deseo de la conducta de juego (puesto que el paciente llegó con control de estímulos y ya no se encontraba jugando); b) establecimiento de balance decisional como técnica para mantener la motivación al cambio y la conducta de abstinencia y c) puesta en marcha del diario de actividades realizado en consulta a modo de terapia de activación para asegurar que ocupara el tiempo libre que le quedaría sin el juego, dada su falta de introspección a la hora de decidir qué actividades le gustaban hacer antes de comenzar a apostar para la organización del tiempo libre sin juego (ver Figura 2). 
Figura 1

Contrato terapéutico

H

nime

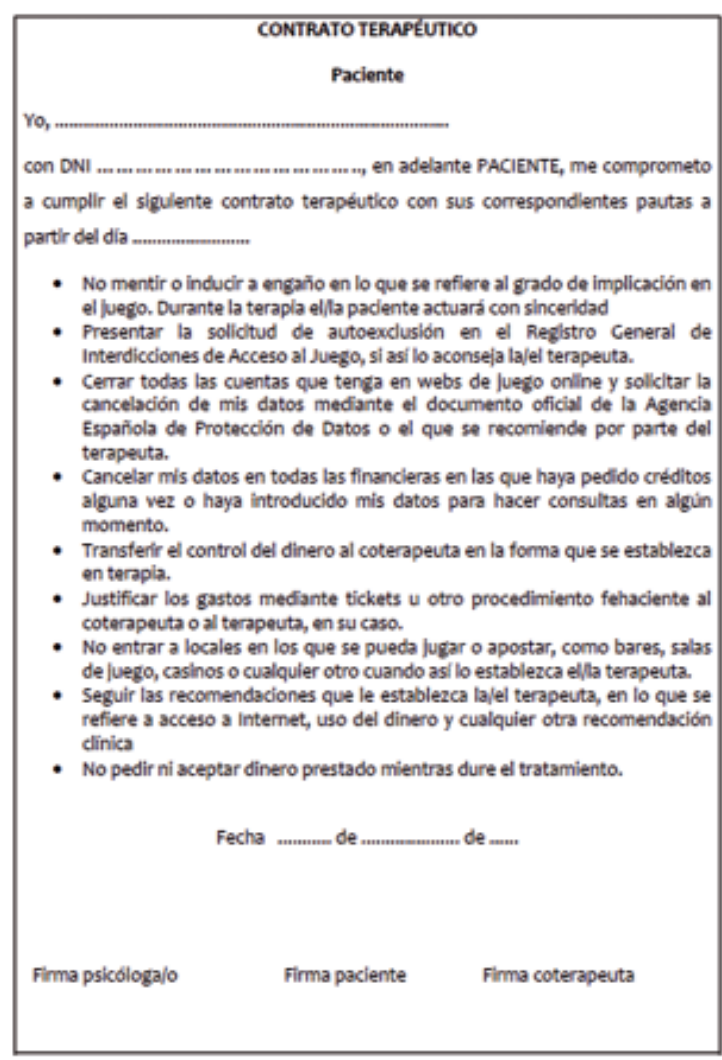

Figura 2

Autorregistro para análisis funcional

\section{\&4}

Autorregistro de juego

Expediente:

\begin{tabular}{|c|c|c|c|c|c|c|c|c|}
\hline $\begin{array}{l}\text { Fechay y } \\
\text { Hora }\end{array}$ & $\begin{array}{l}\text { Tipo de vuego } \\
\text { / lugar }\end{array}$ & $\begin{array}{l}\text { consumo } \\
\text { sustanclas } \\
(\mathrm{A}, \mathrm{Du}, \mathrm{De})\end{array}$ & $\begin{array}{l}\text { Dinero } \\
\text { gastadol } \\
\text { ganodo }\end{array}$ & \begin{tabular}{|l} 
Tiempo \\
dedicado
\end{tabular} & $\begin{array}{l}\text { Que estabos haciendo } \\
\text { ANTES }\end{array}$ & $\begin{array}{l}\text { Qué pensabas / sentias } \\
\text { ANTES }\end{array}$ & $\begin{array}{l}\text { Qué hiciste } \\
\text { DEspuEs }\end{array}$ & $\begin{array}{l}\text { Qué pensaste sentiste } \\
\text { DESPUEs }\end{array}$ \\
\hline & & & & & & & & \\
\hline & & & & & & & & \\
\hline & & & & & & & & \\
\hline & & & & & & & & \\
\hline & & & & & & & & \\
\hline & & & & & & & & \\
\hline & & & & & & & & \\
\hline & & & & & & & & \\
\hline & & & & & & & & \\
\hline
\end{tabular}


Del autorregistro del deseo de juego (ver Figura 3) se observó que los principales desencadenantes fueron los estímulos relacionados con el tenis. Alberto tenía pensamientos recurrentes sobre competiciones de tenis, que le llevaban a consultar constantemente el ranking de la Asociación de Tenis Profesional (ATP) masculino, como el de la Women's Tennis Association (WTA), la clasificación femenina de tenis profesional. Tenía el convencimiento de que era capaz de analizar todo y que sabía de tenis lo suficiente como para ganar dinero con las apuestas. Estaba obsesionado por analizar todas las variables y después apostar a ganar o perder a toda la parrilla de jugadores del torneo. Nunca cerraba las apuestas antes de terminar los partidos porque confiaba plenamente en su experticia. Esta creencia era una fuente de refuerzo positivo que, al ponerse a analizar desde su celular, le hacía sentir eufórico y alegre con pensamientos firmes sobre sí mismo de ser bueno analizando y de tener un gran conocimiento en tenis. Sin embargo, la consecuencia más importante que contribuía a mantener el problema era el alivio de estar solo y sin hacer nada, lo cual le provocaba una gran angustia emocional. De esta manera, el problema se mantenía por refuerzo negativo, que aliviaba una y otra vez a corto plazo con el análisis pormenorizado y la consiguiente apuesta, un hecho que perpetuaba a largo plazo la conducta de juego patológico.

Figura 3

Autorregistro del deseo de jugar

\begin{tabular}{|c|c|c|c|c|c|c|c|c|c|c|c|}
\hline \multicolumn{12}{|c|}{ Autorregistro del Deseo de jugar } \\
\hline Fecchay hors & 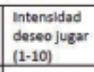 & 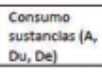 & \begin{tabular}{|l|}
$\begin{array}{l}\text { aud estabosas } \\
\text { hacendo } \\
\text { avres }\end{array}$ \\
\end{tabular} & 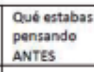 & 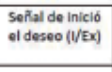 & 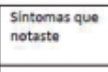 & 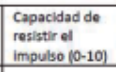 & 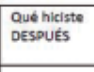 & 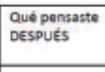 & $\begin{array}{ll}\text { aHas Jugeado? } \\
\end{array}$ & 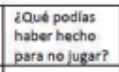 \\
\hline & & & & & & & & & & & \\
\hline & & & & & & & & & & & \\
\hline & & & & & & & & & & & \\
\hline & & & & & & & & & & & \\
\hline & & & & & & & & & & & \\
\hline & & & & & & & & & & & \\
\hline
\end{tabular}

2. Abstinencia y superación del malestar. Los principales objetivos de esta fase fueron los siguientes: a) el mantenimiento de la abstinencia, b) la superación del malestar provocado por la privación del juego y c) el fomento de conductas alternativas que sustituyeran a las apuestas.

Para el mantenimiento de la abstinencia se procedió a reforzar y alentar la continuación de la abstinencia del juego mediante las técnicas de control de estímulo que se diseñaron según el análisis funcional retrospectivo de la conducta. Las medidas acordadas consistieron en transferir el control del dinero a la madre (tarjetas bancarias, transferencias, etc.), limitar la cantidad de dinero de bolsillo cuyos gastos debería justificar mediante recibos, evitar itinerarios que incluyeran salas de apuestas, restringir la utilización del móvil únicamente para responder mensajes de WhatsApp o realizar llamadas, autoexcluirse del juego en la Dirección General de Ordenación de Juego, e inscribirse en el fichero de autoprotección de microcréditos. En esta fase se decidió adelantar la prevención de recaídas informando al paciente de aquellas situaciones de riesgo conocidas dentro de los hallazgos de la investigación científica para evitar la recaída mediante la información veraz y aprovechando la alianza terapéutica. 
Para la superación del malestar se entrenó en relajación muscular progresiva tipo Jacobson (RMP) y técnicas de respiración. La duración de los ejercicios se fue reduciendo y adaptando a las situaciones que le provocaban malestar en contextos naturales.

Por último, se organizaron actividades diarias de ocio y tiempo libre a lo largo de la semana en base a sus preferencias y recursos. La finalidad fue establecer un repertorio de conductas alternativas e incompatibles con el juego que, inicialmente, ayudaran a mantener la abstinencia, pero que fueran la base para promover un posterior estilo de vida alternativo en consonancia con sus principios y su ideal proyecto de vida como meta a alcanzar. La duración de esta fase fue de 3 sesiones.

\section{Descondicionamiento y promoción de un} nuevo estilo de vida. Los objetivos que se persiguieron en esta fase fueron los siguientes: a) descondicionamiento de los antecedentes (estímulos condicionados) asociados al juego y las contingencias del mismo; b) disminución del interés y el agrado que provocan las apuestas; c) adquisición de un estilo de vida saludable alternativo a las apuestas y d) aprendizaje para resistir el impulso de jugar e incluso el entrenamiento en parar una vez que se hubiera iniciado el juego con el objetivo de que no se llegara a apostar aunque se encontrara en una situación de juego. Al procedimiento desarrollado por Echeburúa et al. (1996), consistente en exposición en vivo con prevención de respuesta graduada en base a Unidades Subjetivas de Síndrome de Abstinencia (USSA), se le añadió el entrenamiento en estrategias de afrontamiento. Ello para conseguir no solamente el descondicionamiento de las respuestas condicionadas compensatorias, sino también de la adquisición de las estrategias de afrontamiento eficaces que le fueran de utilidad en las situaciones de riesgo en las que pudiera encontrarse en un futuro (utilización del móvil, webs de apuestas, anuncios publicitarios, etc.). Se establecieron tres niveles de dificultad. Finalmente, el paciente consiguió navegar por webs de juego sin apostar ni sentir las ganas de hacerlo.

La duración de esta fase fueron seis sesiones con un espacio temporal de dos semanas.

Figura 4

Autorregistro para las sesiones de exposición

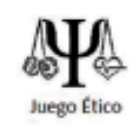

Autorregistro para Exposición

\begin{tabular}{|c|c|c|c|c|c|c|c|}
\hline & Lunes & Martes & Miércoles & Jueves & Viernes & Sábado & Domingo \\
\hline Evento: & & & & & & & \\
\hline $\begin{array}{l}\text {-Deseo de apostar }(0-10) \\
\text {-Ansiedad durante }(0-10) \\
\text {-Ansiedad al acabar }(0-10)\end{array}$ & & & & & & & \\
\hline Evento & & & & & & & \\
\hline $\begin{array}{l}\text {-Deseo de apostar }(0-10) \\
\text {-Ansiedad durante }(0-10) \\
\text {-Ansiedad al acabar }(0-10)\end{array}$ & & & & & & & \\
\hline
\end{tabular}




\section{Prevención de recaídas y consolidación de} nuevo estilo de vida. Los objetivos fueron los siguientes: a) aprender a identificar posteriores situaciones de riesgo que pudieran inducir una conducta de juego y b) aprender a reaccionar apropiadamente ante las mismas. El entrenamiento se llevó a cabo tanto en sesiones individuales (tres sesiones), como en grupo (dos sesiones). En las sesiones individuales se analizaron las dificultades que tuvo antes de iniciar el tratamiento que le condujeron a recaer, así como las acciones que debería haber llevado a cabo en su momento para que no se hubieran producido las recaídas; se trató de un análisis retrospectivo de sus recaídas anteriores. Así también se realizó el análisis de eventuales situaciones que serían posibles que le ocurrieran en el futuro: se le enseñó a detectarlas y se le entrenó en estrategias para reaccionar en dichos casos. En las sesiones grupales se utilizaron técnicas de discusión aprovechando las recaídas ocurridas en otros pacientes en tratamiento por adicción al juego online por considerarse que podrían resultar en un aprendizaje en beneficio del grupo. Además, se propusieron al paciente las siguientes tareas para casa: registro de recaídas anteriores y análisis de metas y valores. Con ello, se pretendió que, una vez recordadas sus potencialidades y fortalezas, pudiera poner en orden sus valores y estableciera objetivos a medio y largo plazo en consonancia con sus principios de vida que otorgarían interés y autodeterminación hacia la dirección marcada.

De forma transversal, y a lo largo de todas y cada una de las fases descritas, se proporcionó información veraz sobre cómo está diseñado el juego de azar, la publicidad y las estrategias de marketing del sector que hacen que el jugador entre en el ciclo de la adicción: depositar efectivo - apostar - depositar efectivo. El objetivo era demostrar que, además de que el juego de azar no es un juego justo porque su esperanza matemática siempre es favorable para quien lo gestiona y desfavorable para los jugadores, la base del negocio es, precisamente, la gente que tiene problemas, puesto que son los que más juegan y, por lo tanto, los que más pierden (Williams \& Wood, 2004). De esta manera, la finalidad fue conseguir en el paciente un cambio de actitudes hacia el juego que eliminara de raíz la creencia falsa de obtener dinero fácil con el juego. A medida que se iba avanzando en la intervención, se repetía que el juego es un negocio en el que la casa siempre gana con las apuestas y que el valor esperado del jugador siempre es negativo, es decir, que el jugador, a la larga, lo que más seguro tiene son las pérdidas (Chóliz \& Marcos, 2018).

\section{Resultados}

Las puntuaciones antes y después del tratamiento se presentan en la Tabla 1.

En la actualidad, después de las 16 sesiones de tratamiento y tras un periodo de seguimiento de un año, Alberto se encuentra bien, en concreto:

- No ha realizado ninguna apuesta y tampoco tiene interés en realizarlas.

- Sigue teniendo interés por las actividades deportivas: practica deporte y ve partidos de tenis y fútbol, pero sin tener deseos de apostar, llegando a sentir indignación cuando ve la publicidad de juego porque es consciente del problema que generan las apuestas en los jóvenes con los que trabaja.

- Han desaparecido todos los criterios de trastorno de juego, medidos con el NODS.

- Tiene un contrato indefinido de trabajo como monitor de deportes en una empresa.

- Se encuentra optimista, con objetivos vitales y profesionales que le ilusionan, lo cual indica que el tratamiento no solamente ha conseguido eliminar la adicción al juego, sino promover un estilo de vida saludable e incompatible con las apuestas. 
Tabla 1

Resultados de los cuestionarios SCL-90 y NODS antes y después del tratamiento

\begin{tabular}{lcc}
\hline & Antes & Después \\
\hline Somatización & 14 & 19 \\
Obsesividad-compulsividad & 32 & 18 \\
Sensibilidad social & 18 & 11 \\
Depresión & 25 & 16 \\
Ansiedad & 14 & 11 \\
Hostilidad & 10 & 6 \\
Ansiedad fóbica & 9 & 10 \\
Ideación paranoide & 7 & 8 \\
Psicoticismo & 10 & 10 \\
IGS & 156 & 117 \\
NODS & 9 & 2 \\
\hline
\end{tabular}

\section{Discusión}

El tratamiento cognitivo conductual es el que se ha demostrado más eficaz para el tratamiento de la adicción al juego (Petry et al., 2006; Sylvain et al., 1997; Tolchard \& Battersby, 2013). El mismo que tiene en cuenta tanto los aspectos puramente conductuales, como la exposición a señales (Ashrafioun et al., 2012; Riley, 2015; Riley et al., 2011; Riley et al., 2018), y los aspectos cognitivos (Smith et al., 2013).

El presente estudio de caso corresponde a un protocolo de intervención conductual-cognitiva basada en los principios del aprendizaje y de la motivación desarrollado por Chóliz y Marcos (2020), dividida en las siguientes etapas:

a) Evaluación y diagnóstico del problema y análisis funcional de la conducta

En esta etapa se pretende tener una comprensión global de la conducta problema, de la evolución que ha tenido el trastorno y los condicionantes personales y ambientales que inducen y mantienen la conducta de juego. b) Abstinencia y superación del malestar y el deseo de jugar

En esta etapa se pretende que la persona deje de jugar, para lo cual se modifican las condiciones que inducen al juego (control del dinero y del uso del celular, autoexclusión de los juegos online y de los microcréditos). Al mismo tiempo, se le suministran herramientas para superar el malestar provocado por la privación mediante la realización de conductas alternativas a las apuestas. Para ayudar a mantener la abstinencia se fomentan conductas que ocupen el tiempo y le resulten gratificantes.

c) Descondicionamiento y promoción de un nuevo estilo de vida

Es una de las fases más relevantes. Consiste en descondicionar los estímulos ambientales y personales que incitan a jugar mediante la extinción de la respuesta condicionada compensatoria de un modo gradual. Para ello, Alberto debía exponerse a situaciones de juego y ser capaz de resistir el impulso el tiempo suficiente para disminuir su deseo de jugar. Durante las 
exposiciones se ayuda con técnicas de autocontrol y en los momentos en los que no está expuesto al juego se fomentan actividades alternativas, muchas de las cuales se han iniciado en la etapa anterior.

Dichas conductas deben ser motivadoras al tiempo que ayuden a consolidar un nuevo estilo de vida sin juego.

d) Prevención de recaídas y consolidación de un nuevo estilo de vida

El tratamiento finaliza una vez el paciente se encuentre entrenado para resolver situaciones de riesgo en las que se pueda ver inmerso, de manera que actúe automáticamente de forma eficaz. Así como también, pueda realizar una evaluación global de los cambios producidos en otras áreas de la vida. En el caso de Alberto fueron la mejora de la economía, la obtención de trabajo remunerado y el establecimiento de actividades de ocio saludables.

En síntesis, el tratamiento propuesto en este estudio de caso se basa en los principios de la psicología del aprendizaje, entendiendo que las conductas adictivas se caracterizan por tratarse de comportamientos estereotipados inducidos por condiciones ambientales o intrapersonales específicas que tienden a repetirse e incluso incrementarse. Su fundamento no solo trata de explicar la génesis y mantenimiento de la adicción al juego bajo el paradigma de condicionamiento clásico, sino también del tratamiento basado en control de estímulo y exposición gradual con prevención de respuesta (Echeburúa et al., 1996). Además, la conducta de juego no aparece en el vacío y está regida por los principios del condicionamiento operante debido a que la acción de apostar tiene como consecuencia la aparición (o no) de una recompensa monetaria, según programas concurrentes de reforzamiento (Pierce \& Cheney, 2013). De esta manera, es preciso que ante la aparición de los estímulos discriminativos que indican que el refuerzo está disponible (y los hay muchos en forma de publicidad, promociones, tipos de apuestas o competiciones, estrategias de marketing, etc.), la persona sepa y sea capaz de abstenerse de jugar, así como de realizar otras conductas alternativas al juego. En este caso, se trata de reducción o eliminación de la conducta mediante la adquisición de estrategias de afrontamiento o de fomento de las alternativas mediante la sustitución conductual (O’Donohue \& Fisher, 2012). Con ello, se pretendió no solamente la eliminación de la conducta desadaptativa, sino también la consolidación de un estilo de vida funcional y saludable.

Finalmente, se promovió que la persona entendiera no solamente la génesis de su problema, sino el fundamento del tratamiento para motivarse lo suficiente como para mantener unas pautas de comportamiento que, aunque costosas de mantener, le suministrarían a su vez información de cómo está organizado el juego socialmente en España.

\section{Conclusiones}

Se ha desarrollado un protocolo de tratamiento de la adicción al juego online basado en los principios de la motivación y el aprendizaje (Chóliz \& Marcos, 2020). Se trata de un modelo de intervención conductualcognitivo que pretende no solo reducir una conducta de juego que está provocando la ruina personal (y esta no solo es económica), sino modificar el estilo de vida y consolidar pautas de comportamiento funcionales para tener una vida plena y sin juego. La intervención no solamente ha demostrado ser eficaz en la reducción de la conducta problema y la generación de alternativas que conforman un estilo de vida saludable, sino que se ha visto reforzada con una actitud de distanciamiento hacia las apuestas y de recursos de afrontamiento de las situaciones críticas que se espera que sirvan para prevenir eventuales recaídas.

El presente estudio de caso no está exento de limitaciones que es necesario tener en cuenta. Se trata de la imposibilidad de tomar la línea base inicial de la conducta de juego de Alberto ya que, cuando llegó a consulta, venía abstinente y con control de estímulos establecido por la madre. Esta casuística 
personal hizo que el análisis funcional del juego se realizara retrospectivamente, confiando en la veracidad de los datos que proporcionó, pero siendo consciente como terapeuta de la necesidad de contrarrestar en cada momento la información dada con los autorregistros de deseo de juego que cumplimentó durante todo el tratamiento con el objeto de ir adecuando el plan de intervención a las necesidades que se iban detectando.

\section{Conflicto de intereses}

La autora declara no tener conflicto de intereses con el sector del juego. Este trabajo no ha sido financiado ni total ni parcialmente por la industria del juego.

\section{Responsabilidad ética}

Se ha respetado la normativa establecida en la Ley Orgánica 3/2018 del 5 de diciembre, de Protección de Datos de Carácter Personal, además de las que figuran en el Real Decreto 5/2018 del 27 de julio. Los procedimientos realizados en este estudio se ajustaron a las normas éticas de la Declaración de Helsinki de 1964 y sus modificaciones posteriores. El protocolo de tratamiento ha sido aprobado por el Ministerio de Sanidad de España en la convocatoria de proyectos para fines de interés social.

Este trabajo ha sido posible gracias a la subvención del Plan Nacional sobre Drogas (PNSD) concedida a la Unión de Asociaciones y Entidades de Atención al Drogodependiente (UNAD) para el desarrollo, desde la Universidad de Valencia, de un protocolo de tratamiento psicológico de la adicción al juego online, en el marco de las subvenciones de 2017 del Ministerio de Sanidad para fines de interés social. Se estableció contrato con la Universidad de Valencia: OTR2018-19316ASESO.

La autora se adscribe al código ético de investigación científica sobre el juego H.E.A.R.T. (Auckland, 2018).

\section{Agradecimientos}

Este estudio ha sido financiado por el Ministerio de Sanidad, Servicios Sociales e Igualdad de España mediante contrato con la Universidad de Valencia: OTR2018-19316ASESO

\section{Referencias}

American Psychiatric Association. (1980). Diagnostic and statistical manual of mental disorders (DSM-III). American Psychiatric Pub.

American Psychiatric Association. (2000). DSM-IV-TR. Barcelona: Masson.

American Psychiatric Association. (2013). Diagnostic and statistical manual of mental disorders (DSM-5). American Psychiatric Pub.

Ashrafioun, L., McCarthy, A., \& Rosenberg, H. (2012). Assessing the impact of cue exposure on craving to gamble in university students. Journal of Gambling Studies, 28(3), 363-375. https://doi.org/10.1007/s10899011-9262-0

Barlow, D. H., \& Hersen, M. (1998). Diseños experimentales de caso único. Martínez Roca.

Chóliz, M. (2018). Ethical Gambling: A Necessary New Point of View of Gambling in Public Health Policies. Frontiers in Public Health, 6, 12. https://doi.org/10.3389/fpubh.2 018.00012

Chóliz, M., \& Marcos, M. (2018). Cuando jugar es un problema. Psylicom Ediciones.

Chóliz, M., \& Marcos, M. (2020). Tratamiento psicológico de la adicción al juego online. Ediciones Pirámide.

Chóliz, M., Marcos, M., \& Lázaro-Mateo, J. (2019). The Risk of Online Gambling. A Study of Gambling Disorder Prevalence Rates in Spain. International Journal of Mental Health and Addiction, 11(4). https://doi.org/ 10.1007/s11469-019-00067-4

Derogatis, L. R., \& Cleary, P. A. (1977). Confirmation of the Dimensional Structure of the SCL-90: A study in construct validation. Journal of Clinical Psychology, 33(4), 981-989. https://doi.org/10.1002/ 1097-4679(197710)33:4<981::AID-JCLP2270330412> 3.0.CO;2-0 
Echeburúa, E., Báez, C., \& Fernández-Montalvo, J. (1996). Comparative Effectiveness of Three Therapeutic Modalities in the Psychological Treatment of Pathological Gambling: Long-Term Outcome. Behavioural and Cognitive Psychotherapy, 24(1), 5172. https://doi.org/10.1017/S1352465800016830

Gerstein, D., Hoffmann, J., Larison, C., Engelman, L., Murphy, S., Palmer, A., Chuchro, L., Toce, M., Johnson, R., Bluie, T., \& Hill, M. A. (1999). Gambling impact and behavior study. Report to the National Gambling Impact Study Commission. National Opinion Research Center at the University of Chicago.

Goudriaan, A., Oosterlaan, J., de Beurs, E., \& Van den Brink, W. (2004). Pathological Gambling: A Comprehensive Review of Biobehavioral Findings. Neuroscience and Biobehavioral Reviews, 28(2), 123-141. https://doi.org/ 10.1016/j.neubiorev.2004.03.001

Griffiths, M. (2003). Internet Gambling: Issues, Concerns, and Recommendations. CyberPsychology \& Behavior, 6(6), 557-568. https://doi.org/10.1089/109493103322725333

Griffiths, M., \& Barnes, A. (2008). Internet Gambling: An Online Empirical Study Among Student Gamblers. International Journal of Mental Health and Addiction, 6(2), 194-204. https://doi.org/10.1007/s11469-007-9083-7

Marks, I. (1990). Behavioural (non-chemical) addictions. British journal of addiction, 85(11), 1389-1394. https: //doi.org/10.1111/j.1360-0443.1990.tb01618.x

McBride, J., \& Derevensky, J. (2012). Internet Gambling and Risk-Taking Among Students: An Exploratory Study. Journal of Behavioral Addiction, 1(2), 50-58. https:// doi.org/10.1556/jba.1.2012.2.2

Miller, W., \& Rollnick, S. (1991). La entrevista motivacional. Preparar para el cambio de conductas adictivas. Ediciones Paidós Ibérica, S. A.

Montero, I., \& León, O. G. (2005). Sistema de clasificación del método en los informes de investigación en Psicología. International Journal of Clinical and Health Psychology, 5(1), 115-127.

O’Donohue, W. T., \& Fisher, J. E. (eds.). (2012). Cognitive Behavior Therapy: Core Principles for Practice. John Wiley \& Sons.

Organización Mundial de la Salud - OMS. (1977). Clasificación Internacional de las Enfermedades (9. ${ }^{a}$ ed.). https://www.mscbs.gob.es/estadEstudios/estadis ticas/docs/CIE9MC_2014_def_accesible.pdf

Organización Mundial de la Salud - OMS. (2018a). CIE11. Clasificación Internacional de las Enfermedades 11. ${ }^{\text {a }}$ revisión. https://icd.who.int/es

Organización Mundial de la Salud - OMS. (2018b). ICD11. International Classification of Diseases $11^{\text {th }}$ revision. https://icd.who.int

Petry, N. M., Ammerman, Y., Bohl, J., Doersch, A., Gay, H., Kadden, R., Molina, C., \& Steinberg, K. (2006). Cognitive-behavioral therapy for pathological gamblers. Journal of Consulting and Clinical Psychology, 74(3), 555-567. http://dx.doi.org/10.1037/ 0022-006X.74.3.555

Pierce, W. D., \& Cheney, C. D. (2013). Behavior Analysis and Learning ( $5^{\text {th }}$ ed.). Psychology Press. https:// doi.org/10.4324/9780203441817

Prochaska, J. O., DiClemente, C. C., \& Norcross, J. C. (1992). In Search of How People Change: Applications to Addictive Behaviors. American Psychologist, 47(9), 1102-1114. https://doi.org/10.1037/0003-066X.47.9.1102

Riley, B. J. (2015). The Role of Homework in Exposure-Based CBT Outcome for Problem Gambling. International Gambling Studies, 15(3), 394-407. https://doi.org/ 10.1080/14459795.2015.1062532

Riley, B., Baigent, M. F., Harris, S., Larsen, A., Nye, T., \& Battersby, M. W. (2018). Extinction of Gambling CueReactivity: A Pilot Study in a Problem Gambling Treatment Setting. http://doi.org/10.33582/2637-4528/ 1010

Riley, B., Smith, D., \& Oakes, J. (2011). Exposure Therapy for Problem Gambling in Rural Communities: A Program Model and Early Outcomes. Australian Journal of Rural Health, 19(3), 142-146. https://doi.org/10.1111/ j.1440-1584.2011.01199.x

Robles, J. I., Andreu, J. M., \& Peña, M. E. (2002). SCL-90: Aplicación y análisis de sus propiedades psicométricas en una muestra de sujetos clínicos españoles. Psicopatología Clínica, Legal y Forense, 2(1), 1-19. https://dialnet.unirioja.es/servlet/articulo?codigo= 2518060

Schüll, N. D. (2014). Addiction by Design: Machine Gambling in Las Vegas. Princeton University Press. 
Smith, D. P., Battersby, M. W., Harvey, P. W., Pols, R. G., \& Ladouceur, R. (2013). Two-Group Randomised, Parallel Trial of Cognitive and Exposure Therapies for Problem Gambling: A Research Protocol. BMJ Open, 3(6). http:// dx.doi.org/10.1136/bmjopen-2013-003244

Sylvain, C., Ladouceur, R., \& Boisvert, J. (1997). Cognitive and Behavioral Treatment of Pathological Gambling: A Controlled Study. Journal of Consulting and Clinical Psychology, 65(5), 727-732. http://dx.doi.org/10.1037/ 0022-006X.65.5.727
Tolchard, B., \& Battersby, M. W. (2013). Cognitive Behaviour Therapy for Problem Gamblers: A Clinical Outcomes Evaluation. Behaviour Change, 30(1), 12-23. https://doi.org/10.1017/bec.2013.2

Williams, R. J., \& Wood, R. T. (2004). The Proportion of Gaming Revenue Derived from Problem Gamblers: Examining the Issues in a Canadian Context. Analyses of Social Issues and Public Policy, 4(1), 33-45. https://doi.org/10.1111/j.1530-2415.2004.00033.x

\section{Marta Marcos Moliner}

Departamento de Psicología Básica, Universidad de Valencia, España

Personal investigador. Psicóloga Sanitaria. Su trabajo se centra en evaluación, prevención y tratamiento de la adicción al juego y a las tecnologías.

ORCID: https://orcid.org/0000-0003-1781-2411

marta.marcos@uv.es 